\title{
Efeito do laser de baixa intensidade no tratamento da disfunção temporomandibular: Relato de caso
}

\author{
Effect of low-level laser therapy in the treatment of temporomandibular disorder: Case report
}

Efecto del láser de baja intensidad en el tratamiento de la disfunción temporomandibular: Informe de caso

\author{
Hortência Barroso Soares Borba \\ ORCID: https://orcid.org/0000-0002-9325-4936 \\ Universidade Federal de Alagoas, Brasil \\ E-mail: hortencia.barroso@hotmail.com \\ Natália Elias da Silva \\ ORCID: https://orcid.org/0000-0002-2135-3564 \\ Universidade Federal de Alagoas, Brasil \\ E-mail: nataaliaelias@gmail.com \\ Stela Maris Wanderley Rocha \\ ORCID: https://orcid.org/0000-0002-3220-648X \\ Universidade Federal de Alagoas, Brasil \\ E-mail: stelawanderley@ hotmail.com \\ Ricardo Viana Bessa Nogueira \\ ORCID: https://orcid.org/0000-0002-9776-6752 \\ Universidade Federal de Alagoas, Brasil \\ E-mail: ricardo.bessa@foufal.ufal.br
}

\begin{abstract}
Resumo
A disfunção temporomandibular (DTM) é uma patologia multifatorial caracterizada, na maioria dos casos, por condições álgicas que acometem a articulação temporomandibular e/ou os músculos orofaciais. Existem várias modalidades terapêuticas conservadoras, incluindo a laserterapia de baixa intensidade ou LLLT, que são usadas com sucesso para o manejo da DTM. O objetivo deste trabalho foi relatar o caso de uma paciente com disfunção temporomandibular de origem muscular cuja abordagem terapêutica foi a laserterapia e acompanhamento psicológico. A paciente selecionada foi submetida a um protocolo de laserterapia de 12 sessões com um laser diodo de AsGaAl $\left(808 \mathrm{~nm}, 80 \mathrm{~mW}, 120 \mathrm{~J} / \mathrm{cm}^{2}\right.$, contínuo). O instrumento RDC/TMD eixo I e II foi utilizado para avaliar inicialmente a paciente. As variáveis: abertura máxima de boca (medida em $\mathrm{mm}$ ) e dor (mediada pela Escala visual analógica (EVA) 0-10; sendo 0 sem dor e 10 máxima dor) foram analisadas durante as sessões e ao término do tratamento. De acordo com a avaliação pela EVA observou-se uma redução da dor de 8 (pré-irradiação) para 1 (pós-irradiação), e melhora da abertura de boca de $4 \mathrm{~mm}$. Houve um resultado positivo quanto à diminuição da dor miofascial e melhora na abertura de boca devido à disfunção apresentada pela paciente. Por conseguinte, a laserterapia mostra-se uma opção viável no controle da DTM de origem muscular.
\end{abstract}

Palavras-chave: Articulação temporomandibular; Lasers; Terapia a laser; Dor facial; Transtornos da articulação temporomandibular.

\begin{abstract}
Temporomandibular disorder (TMD) is a multifactorial pathology characterized, in most cases, by pain conditions that affect the temporomandibular joint and / or the orofacial muscles. There are several conservative therapeutic modalities, including low-level laser therapy or LLLT, which are used successfully for the management of TMD. The aim of this study was to report the case of a patient with temporomandibular disorder of muscular origin whose therapeutic approach was laser therapy and psychological monitoring. The selected patient was submitted to a 12session laser therapy protocol with an AsGaAl diode laser $\left(808 \mathrm{~nm}, 80 \mathrm{~mW}, 120 \mathrm{~J} / \mathrm{cm}^{2}\right.$, continuous). The RDC / TMD axis I and II instrument was used to initially assess the patient. The variables: maximum mouth opening (measured in $\mathrm{mm}$ ) and pain (mediated by the Visual Analogue Scale (VAS) 0-10; 0 with no pain and 10 with maximum pain) were analyzed during the sessions and at the end of treatment. According to the evaluation by EVA, there was a reduction in pain from 8 (pre-irradiation) to 1 (post-irradiation), and an improvement in mouth opening of $4 \mathrm{~mm}$. There was a positive result regarding the decrease in myofascial pain and improvement in the opening of the mouth due to the dysfunction presented by the patient. Therefore, laser therapy is a viable option in the control of TMD of muscular origin.
\end{abstract}

Keywords: Temporomandibular joint; Lasers; Laser therapy; Facial pain; Temporomandibular joint disorders. 


\section{Resumen}

El trastorno temporomandibular (TMD) es una patología multifactorial caracterizada, en la mayoría de los casos, por cuadros dolorosos que afectan la articulación temporomandibular y / o los músculos orofaciales. Existen varias modalidades terapéuticas conservadoras, incluida la terapia con láser de bajo nivel o LLLT, que se utilizan con éxito para el tratamiento de TMD. El objetivo de este estudio fue reportar el caso de un paciente con trastorno temporomandibular de origen muscular cuyo abordaje terapéutico fue la terapia láser y el seguimiento psicológico. El paciente seleccionado fue sometido a un protocolo de terapia láser de 12 sesiones con un láser de diodo $\mathrm{AsGaAl}(808$ $\mathrm{nm}, 80 \mathrm{~mW}, 120 \mathrm{~J} / \mathrm{cm}^{2}$, continuo). Se utilizó el instrumento RDC / TMD de los ejes I y II para evaluar inicialmente al paciente. Las variables: máxima apertura bucal (medida en $\mathrm{mm}$ ) y dolor (mediado por la Escala Visual Analógica (EVA) 0-10; 0 sin dolor y 10 con dolor máximo) se analizaron durante las sesiones y al final del tratamiento. Según la evaluación de EVA, hubo una reducción del dolor de 8 (preirradiación) a 1 (posirradiación) y una mejora en la apertura de la boca de $4 \mathrm{~mm}$. Hubo un resultado positivo en cuanto a la disminución del dolor miofascial y mejoría en la apertura de la boca debido a la disfunción que presenta el paciente. En consecuencia, la terapia con láser es una opción viable en el control de TMD de origen muscular.

Palabras clave: Articulación oreja-mandíbula; Láseres; Terapia con láser; Dolor facial; Trastornos de la articulación temporomandibular.

\section{Introdução}

Disfunção temporomandibular (DTM) é um termo que abrange várias alterações clínicas envolvendo a musculatura mastigatória, as articulações temporomandibulares (ATM) e estruturas associadas. (Bessa-Nogueira, Vasconcelos \& Niederman, 2008; Castillo, Azato, Coelho, Pereira \& Silva, 2016; Dantas, Santos, Vilela, \& Lucena, 2015). Os sintomas mais comuns da DTM incluem dores na ATM ou na musculatura associada, limitação de abertura, estalidos, crepitação ao abrir ou fechar a boca, disfunção articular, otalgia, zumbidos, tonturas, dor de cabeça e no pescoço (Castillo et al., 2016; Dantas et al., 2015; Pozzebon, Piccin, Silva \& Corrêa, 2016). A etiologia da DTM é considerada complexa e multifatorial, visto que os fatores que a desencadeia podem ser físicos, psicológicos, traumáticos ou patológicos (Castillo et al. (2016); Dantas et al., 2015).

O tratamento é baseado em um correto diagnóstico obtido pela identificação e levantamento de sinais e sintomas, do estabelecimento de possíveis fatores etiológicos, tempo de instalação do problema, sintomatologia clínica e nível de comprometimento das estruturas musculares e articulares (Venancio, Camparis, \& Lizarelli, 2002; Catão, Oliveira, Costa, \& Carneiro, 2012). As terapias conservadoras — termoterapia, ultrassom, agentes analgésicos e a laserterapia — são capazes de propiciar aos pacientes alívio dos sintomas nas DTMs de origem muscular. (Quinto, 2000).

A laserterapia de baixa intensidade (Low-level Laser Therapy - LLLT) foi introduzida por Endre Mester no final da década de 60, na Hungria e tem sido utilizada como tratamento primário nas disfunções temporomandibulares (Dias et al. 2009; Salmos-Brito et al. 2013) É um procedimento não invasivo, fácil de ser empregado, com custo benefício excelente para profissional e paciente, e não possui contraindicações Catão et al. (2012). Dentre os efeitos que desempenha destacam-se a aceleração de processos cicatriciais, modulação da inflamação e analgesia, permitindo melhora da abertura bucal e da mastigação (Salmos-Brito et al. 2013). Está indicada na presença de dor, restrições de movimentos mandibulares, inflamação do tecido e instabilidade articular (Salmos-Brito et al. 2013; Catão et al. 2012)

Quando se incide radiação eletromagnética sobre um tecido ocorre quatro fenômenos: reflexão, absorção, difusão e transmissão. O mecanismo de ação do laser se dá através da absorção, difusão e transmissão. As células possuem moléculas chamadas cromóforos ou fotorreceptores, que são responsáveis pela absorção da luz laser e a partir daí geram os efeitos que o laser desempenha, efeitos fotoquímicos, fotofísicos e fotobiológicos. (Cavalcanti, Almeida-Barros, Catão, Feitosa, \& Lins, 2011; Henriques, Cazal, \& Castro, 2010) Dentre esses efeitos, existem os que ocorrem durante a emissão do laser, que são os efeitos primários e os que ocorrem mais tardiamente, depois de cessada a irradiação, que são os efeitos secundários. Dentre os primários destacam-se: aumento da produção do metabolismo celular, aumento na produção de ATP, aumento da produção de 
endorfinas endógenas e a diminuição da liberação de transmissores nociceptivos, como a serotonina e a bradicinina. Nos efeitos secundários, têm-se o aumento do fluxo sanguíneo e o aumento da drenagem linfática (Henriques et al. 2010).

Diante do exposto, o presente estudo visa descrever uma abordagem conservadora para tratamento da DTM com o uso da laserterapia em uma paciente que foi submetida a um protocolo específico de irradiação.

\section{Metodologia}

A metodologia utilizada foi inicialmente através de uma entrevista semiestruturada com o paciente e, para complementação dos dados, foi feito levantamento do prontuário médico-odontológico, exames físicos, laboratoriais e imagiológicos. Este trabalho é um relato de caso único, pois possui a intenção de investigar uma realidade e a compreender de forma integral, objetivando o desenvolvimento de conhecimento técnico-científico. Caracteriza-se como uma pesquisa qualitativa, em que serão apresentadas as considerações de teóricos, contemplando os pontos positivos e os negativos em suas respectivas análises. A obtenção de dados e interpretação destes são os focos principais. (Ludke \& Andre, 2013) A paciente assinou o termo de Consentimento Livre e Esclarecido, permitindo a divulgação do caso, a realização de registros em prontuários e que fossem relatadas em supervisões os acontecimentos dos atendimentos, como também declarando que foi devidamente informada quanto ao tratamento que seria submetida.

\section{Relato de Caso}

Paciente MFCS, gênero feminino, 45 anos, melanoderma, compareceu a clínica de DTM e dor orofacial da Universidade Federal de Alagoas, queixando-se de dor forte e intermitente do tipo queimação na hemiface esquerda, dormência na região infraorbitária esquerda e no braço esquerdo, como também, estalido unilateral esquerdo, dor no ouvido, zumbido bilateral, vertigem e cefaleia. De acordo com a história médica atual, a paciente não relatou ser portadora de nenhuma doença e nem estar fazendo uso de medicamentos

Durante os exames físicos, foi avaliada a abertura de boca por meio de paquímetro manual, constatando-se $38 \mathrm{~mm}$. Observou-se padrão de abertura reto e presença de estalidos durante os movimentos de abertura e fechamento, exceto durante protrusão mandibular. Não apresentou nenhuma má-oclusão, apenas perda de alguns elementos dentários superiores e inferiores (Figura 1). A dor e tensão muscular foram avaliadas através de palpação unilateral com pressão firme, feitas por avaliador padronizado, dos músculos temporal posterior, médio e anterior; masseter superior, médio e inferior; trapézio, esternocleidomastoideo, suboccipitais, e sobre a ATM. A paciente referiu dor durante a palpação, que irradiavam para o ouvido e região cervical, e foram verificados pontos-gatilho apenas no masseter esquerdo. 


\section{Figura 1 - Radiografia Panorâmica.}

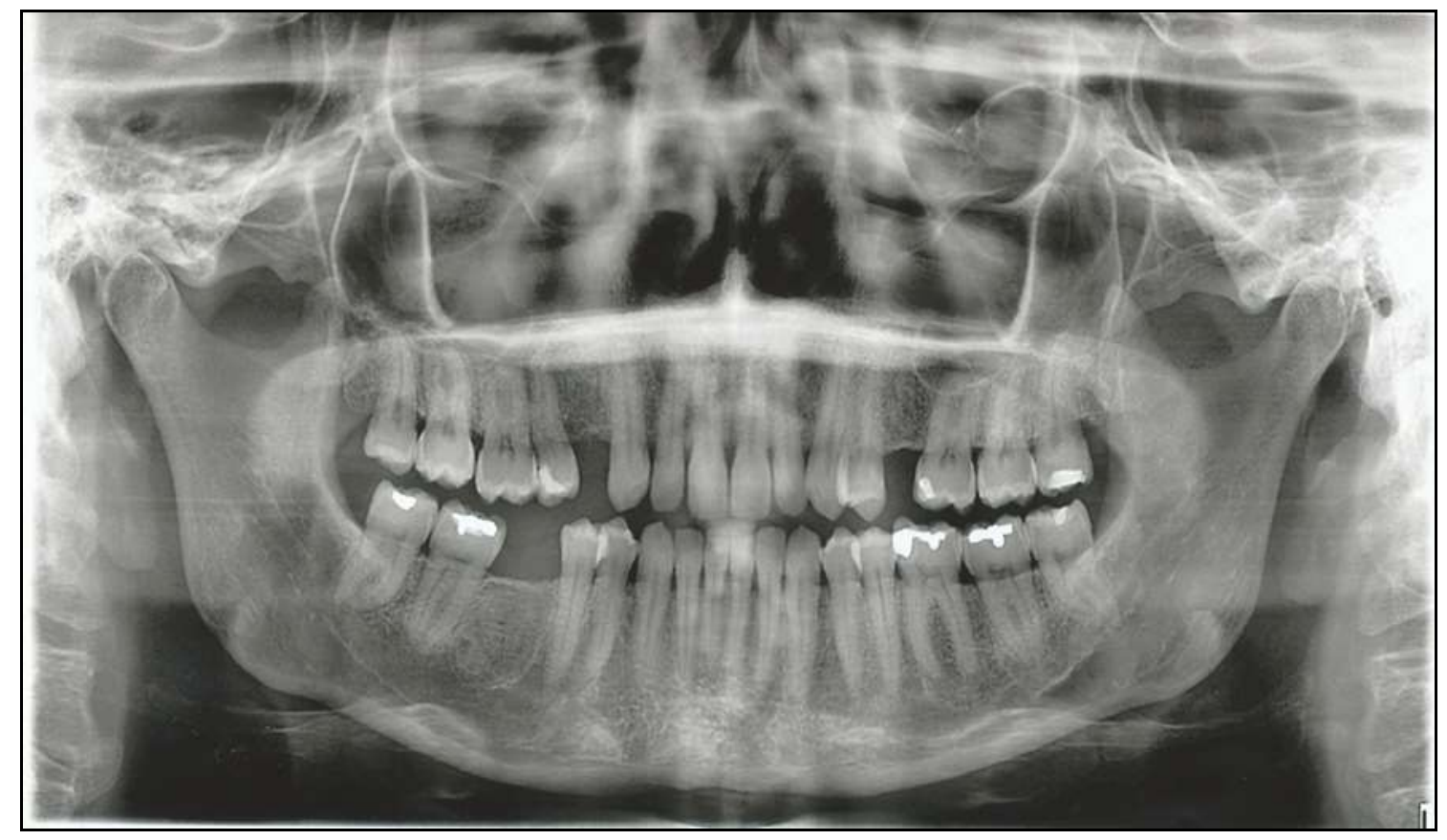

Fonte: Arquivo pessoal dos autores.

O Critério Diagnóstico para Pesquisa em Disfunção Temporomandibular (RDC/TMD) eixos I e II foi utilizado para diagnóstico da disfunção, e avaliação da intensidade da dor crônica, além dos níveis de sintomas depressivos. A identificação dos sinais e sintomas por meio da anamnese, exame físico completo, questionário RDC/TMD eixos I e II, e análise dos exames solicitados, permitiram estabelecer o diagnóstico de DTM muscular e estabeleceu-se então o tratamento com laserterapia de baixa intensidade e acompanhamento psicológico. Através da Escala visual analógica (EVA), inicialmente esclarecida a paciente e aplicada no início e no final de cada sessão de laserterapia, a dor pôde ser avaliada de forma quantitativa. A EVA é uma escala numérica usada, universalmente, que permite quantificar a dor em diversas patologias, incluindo a DTM. Consiste em uma linha horizontal que marca escores de 0 a 10, em que o zero representa "ausência de dor" e o dez a "pior dor possível".

No caso, foi utilizado o laser terapêutico odontológico diodo AsGaAl Whitening Lase II da DMC emitindo irradiação infravermelha, com comprimento de onda de $808 \mathrm{~nm}$, potência de $80 \mathrm{~mW}$, sistema de entrega do feixe por contato direto com a pele, área de focalização de $0,20 \mathrm{~cm}^{2}$, e a dosimetria total de $120 \mathrm{~J} / \mathrm{cm}^{2}$ no tempo de 40 segundos por ponto, de forma pontual. O número de aplicações foi de duas vezes por semana, durante seis semanas totalizando doze aplicações. A psicoterapia foi realizada durante as seis semanas de tratamento, sendo uma sessão por semana totalizando 6 sessões de terapia.

\section{Resultados e Discussão}

Segundo Greenspan e Winfield (1992) dores de cabeça e parestesia unilateral são achados comumente encontrados em pacientes portadores de tumores intracranianos. A paciente do presente estudo apresentou dor na região temporal anterior e dormência na região infraorbitária e braço esquerdo, que podem ser considerados fortes indícios para um tumor cerebral. Por esse motivo, para diagnóstico diferencial, foi solicitada tomografia computadorizada de crânio, hemograma completo e avaliação por um neurologista que descartou as hipóteses de um possível tumor intracraniano. De acordo com Nóbrega (2007) uma investigação criteriosa em casos como esse é indispensável, pois há uma incidência entre 1 e $2 \%$ de pacientes portadores de tumores malignos que foram inicialmente diagnosticados erroneamente, como casos de disfunções temporomandibulares, onde a dor era a queixa principal. 
O laudo da Tomografia Computadorizada descartou as hipóteses de tumor, mas confirmou a presença de cistos de retenção mucosos nos seios maxilares, vistos anteriormente na panorâmica, que de acordo com Carvalho, Vieira e Oliveira (2014), são cistos de caráter benigno e com etiologia multifatorial, podendo estar associado a infecções, alergias ou sinusite. Geralmente são assintomáticos, mas há casos em que são relatados desconforto na região maxilar e hipersensibilidade no terço médio da face, podendo também, a depender de suas dimensões, comprimir o nervo infraorbital e causar parestesias na região por ele inervada, como ocorreu com o caso dessa paciente. Carvalho et al. (2014) ainda diz que na maioria dos casos não se opta por nenhum tratamento, uma vez que a remissão completa do cisto, muitas vezes ocorre de forma espontânea. (Figura 2)

Figura 2 - Tomografia Computadorizada de Crânio.

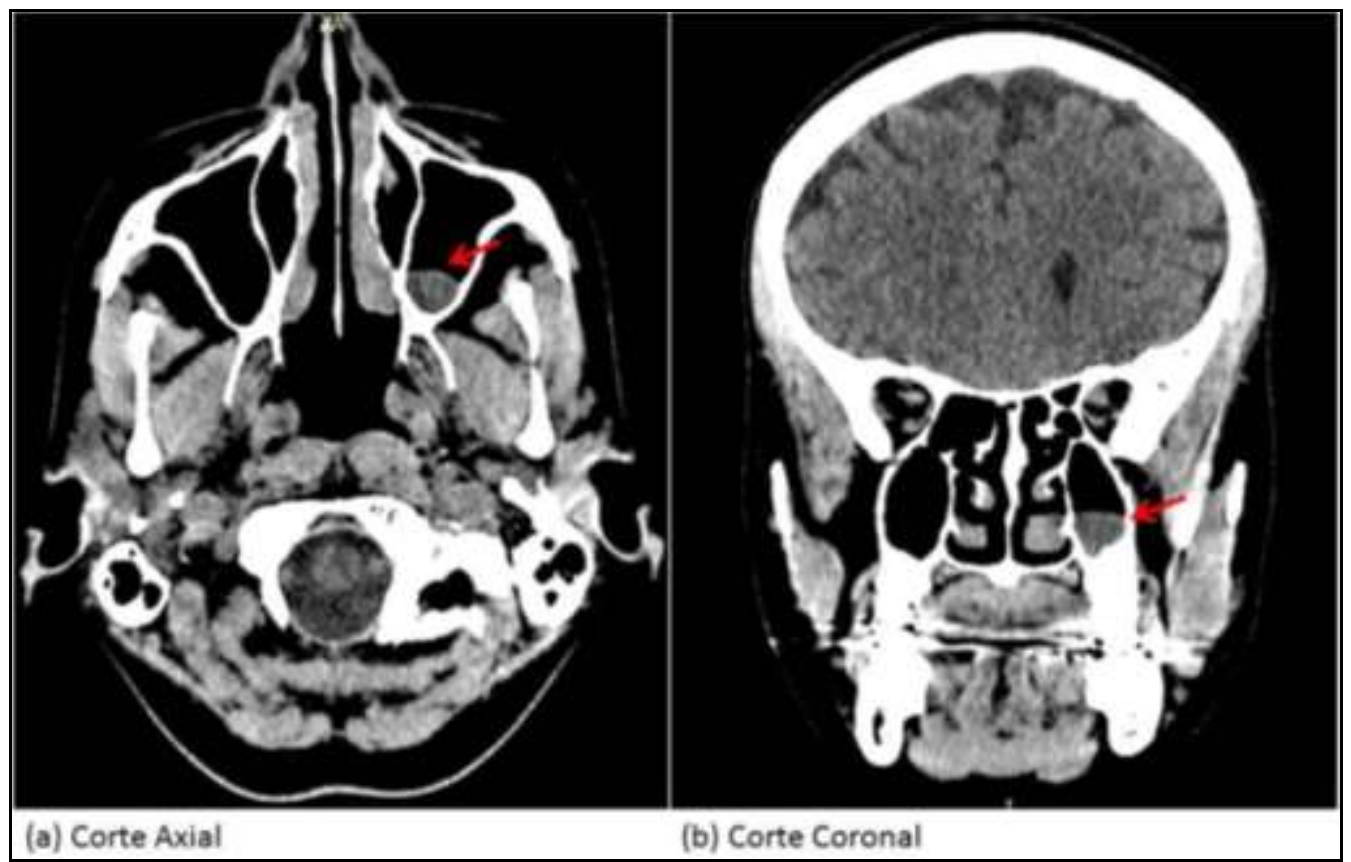

Fonte: Arquivo pessoal dos autores.

O zumbido foi investigado por um otorrinolaringologista e foi descartada a possibilidade de que os sintomas seriam provenientes de problemas de origem otológica, como otites, tumores ou lesões dos órgãos do ouvido. Segundo, Webster, Ikino, Salles, Lino, Manoel e Filho (2011) e Vera, Grillo, Fortinguerra, Sousa e Berzin (2013) o zumbido e a DTM estão em interrelação, pois em portadores da disfunção a presença do zumbido é observada em mais de 50\% dos casos, o que pode ser explicado pela íntima relação anatômica entre o aparelho auditivo e o aparelho estomatognático, dessa forma, o zumbido pode ser um sintoma otológico da DTM (Catão et al., 2012; Vera et al., 2013).

A etiologia da DTM pode estar relacionada com fatores psicológicos como estresse, ansiedade e depressão, influenciando o desenvolvimento de hábitos comportamentais, tais como bruxismo e apertamento dos dentes Castillo et al. (2016), pois as tensões emocionais sobrecarregam os músculos da mastigação, por meio da contração dos mesmos, como afirma Pozzebon et al. (2016). Durante a anamnese, a paciente relatou alto nível de estresse relacionado ao trabalho, o que levava a um sono irregular e má qualidade de vida. Segundo avaliação psicológica, ao final das sessões de psicoterapia, a paciente relatou uma melhora das tensões emocionais, consequente de uma grande diminuição do nível de estresse, corroborando com Piccin et al. (2016) e Friedman (1993), que afirmam que eliminar o fator causal é muito importante, uma vez que esses fatores influenciam na resposta do paciente ao tratamento. 
Além disso, Catão et al. (2012) e Piccin et al. (2016) dizem também que a laserterapia não tem efeito na etiologia ou causa da desordem, portanto os fatores etiológicos devem ser diagnosticados e eliminados para o sucesso do tratamento a longo prazo. Desta forma, a psicoterapia como tratamento coadjuvante auxiliou nos bons resultados obtidos.

De acordo com Dworkin e Leresche (1992) a dor e a restrição de abertura bucal são consideradas os principais sinais clínicos da disfunção temporomandibular. Catão et al. (2012) e Friedman (1993) ressaltam que em adultos a média do limite máximo da abertura mandibular pode variar de 40 a $60 \mathrm{~mm}$, é menor nas mulheres e pode diminuir com a idade. A paciente do caso clínico relatado apresentava no início do tratamento $38 \mathrm{~mm}$ de abertura máxima voluntária, e no final do tratamento estava com $42 \mathrm{~mm}$. Essa não era a principal queixa da paciente, mas a melhoria no presente recurso pode ser entendida como um efeito secundário à redução da dor, assim como visto no estudo de Catão et al. (2012).

Semelhantemente ocorreu com a escala visual analógica (EVA), a dor no início do tratamento era de 8 e após o tratamento diminuiu para 1, com uma melhora de $87,5 \%$. A porcentagem obtida foi realizada por meio da diferença da EVA inicial e EVA final, assim como Vera et al. (2013) (Gráfico 1).

Gráfico 1 - Melhora da intensidade da dor orofacial antes e depois de todas as sessões de laserterapia.

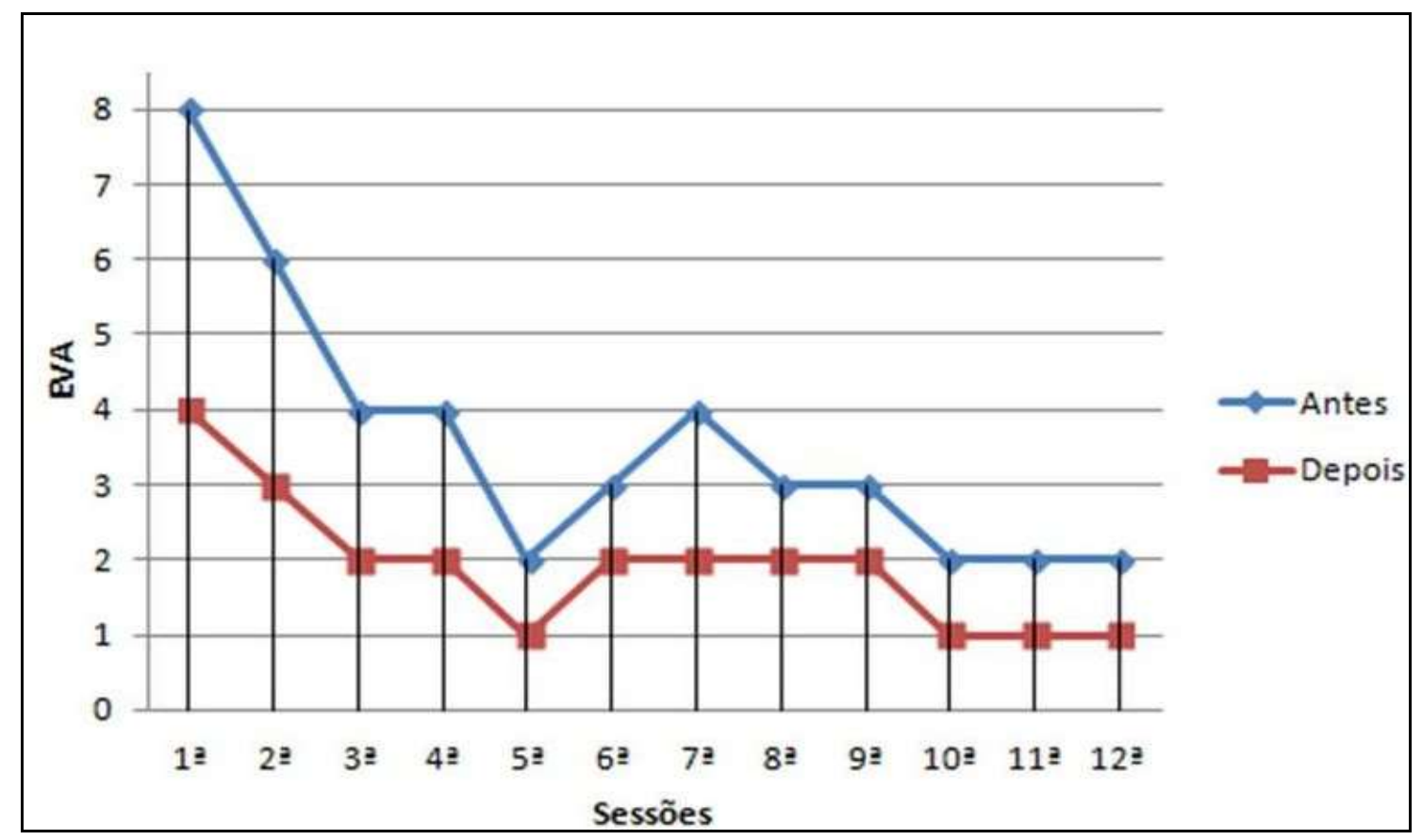

Fonte: Arquivo pessoal dos autores.

Segundo Battistella et al. (2016) os ruídos articulares são um dos principais sinais que acometem os portadores de DTM e de acordo com Lopez (1986) se manifestam em mais de 70\% dos casos. Catão et al. (2012) constataram que a maioria dos pacientes da sua pesquisa apresentavam ruídos, sendo mais prevalentes os estalidos. A paciente do presente estudo apresentou estalido unilateral esquerdo que não mostrou melhora ao final do tratamento com a laserterapia.

A utilização do laser de baixa potência é uma alternativa de tratamento não invasivo, que tem sido amplamente utilizada para o alívio de dor e regeneração tecidual, alcançando resultados satisfatórios, segundo Catão et al. (2012) e explicada no estudo de Henriques et al. (2010) por possuir a capacidade de biomodular a inflamação articular e muscular, promovendo redução da dor ao mesmo tempo em que reduz a contração muscular. Cavalcanti et al. (2011) concorda com os outros autores e conclui que o resultado disso é uma analgesia articular e muscular, melhora da abertura bucal e maior conforto durante a mastigação. 
Ainda segundo Catão et al. (2012) a terapia com laser em portadores de DTM tem demonstrado uma capacidade de alívio das dores em minutos após sua aplicação, o que também foi constatado durante o tratamento do caso apresentado, onde a paciente relatou melhora imediata após todas as sessões. Aparicio, Delgado, Domìnguez, Tosti e Escoda (2013) concluiu, em sua revisão de literatura, que a eficácia clínica do laser de baixa intensidade é controversa, visto que alguns autores obtiveram melhores resultados clínicos em pacientes submetidos ao tratamento com o laser, ao passo que outros não encontraram diferenças significantes. Contudo, quanto ao alívio da dor o presente trabalho observou resultados positivos, confrontando com os diferentes resultados encontrados por Mcneely, Olivo e Magee (2006).

Quanto ao protocolo clínico que deve ser utilizado, Venancio et al. (2002) e Aparicio et al. (2013) dizem que ainda não há um consenso na literatura quanto à intensidade, tempo de exposição e local de aplicação do laser. Nesse caso, as aplicações foram realizadas de forma pontual, em cinco pontos ao redor da articulação, sendo um sobre o côndilo, $1 \mathrm{~cm}$ acima do côndilo, $1 \mathrm{~cm}$ abaixo, $1 \mathrm{~cm}$ anteriormente e $1 \mathrm{~cm}$ posteriormente ao côndilo, e um na região retrodiscal (incidência intra auricular). Seguindo o protocolo utilizado por Catão et al. (2012) aplicou-se no músculo masseter sobre os pontos gatilhos, sendo dois pontos horizontais com $1 \mathrm{~cm}$ de equidistância. (Figura 3).

Figura 3 - (a) Laser terapêutico odontológico diodo AsGaAl Whitening Lase II da DMC; (b) Pontos de aplicação do laser; (c) Distância de 1cm entre os pontos de aplicação do laser; (d) Aplicação do laser de forma pontual.

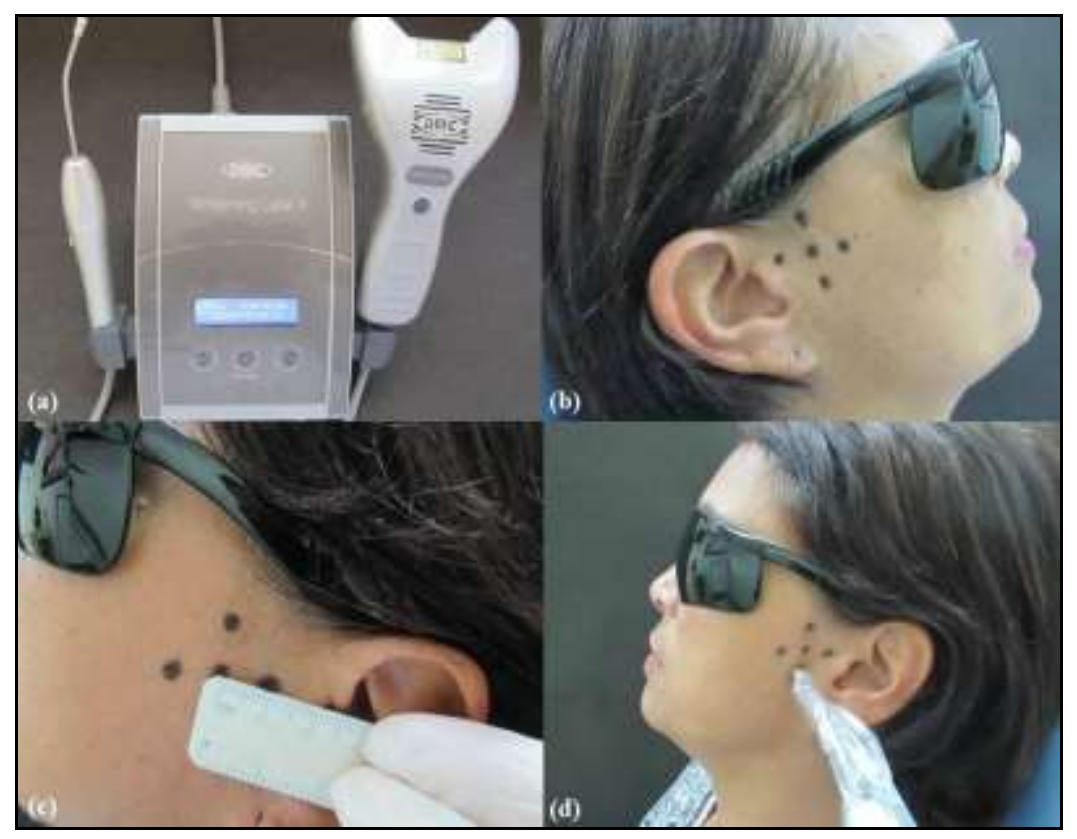

Fonte: Arquivo pessoal dos autores.

As doses por ponto são variáveis, como disse Aparicio et al. (2013) não há consenso na literatura quanto a dose de energia que deve ser aplicada. Henriques et al. (2010) afirmam que uma quantidade de energia entre $1 \mathrm{~J}$ a $2 \mathrm{~J}$ induz um efeito bioestimulatório positivo, o que seria eficaz para a desordem de origem miogênica, já Venâncio et Al. (2002) afirma que a dose de aplicação varia de 6 a $10 \mathrm{~J}$. Segundo as recomendações do fabricante do laser em questão (DMC), a dose de energia ideal para redução da dor varia de 1,7 a 3,5 J, enquanto que a fluência varia de 60 a $120 \mathrm{~J} / \mathrm{cm}^{2}$. Na paciente do estudo a dosimetria por ponto foi em média $3,5 \mathrm{~J}$, com fluência de $120 \mathrm{~J} / \mathrm{cm}^{2}$. A frequência de irradiação também varia de 2 a 5 vezes por semana Venancio et al. (2002). De acordo com Venancio et al. (2002), os lasers emitindo infravermelho são os mais indicados devido à sua maior penetrabilidade, com o espectro eletromagnético de 780 a 904nm, o Whitening Lase II foi utilizado com comprimento de onda de $808 \mathrm{~nm}$. 
As alterações da quantificação da dor através da escala visual analógica (EVA) durante o tratamento com a laserterapia evidenciou a complexidade da disfunção temporomandibular apontada por Catão et al. (2012), uma vez que esta sofre influência de diferentes fatores, dentre os quais estão os psicoemocionais e as atividades exercidas pelo indivíduo durante todo o tratamento. De acordo com Aparicio et al. (2013), a resposta do paciente ao tratamento não depende apenas do tipo de laser, mas também do tecido alvo e das condições do sistema imunológico, da idade do paciente, cor da pele e estágio da patologia (aguda ou crônica). A obtenção de resultados insatisfatórios pode ser decorrente de fatores como: diagnóstico incorreto, doses fracas ou muito elevadas, densidade de energia, quantidade de sessões, dentre outros (ASSIS TO, et al. 2012) Em contrapartida Venancio et al. (2002) cita apenas fatores inerentes ao laser (composição, densidade de potência, quantidade de energia) e fatores relacionados ao tecido (composição protoplasmática celular) como os responsáveis pelas respostas teciduais ao uso da laserterapia.

\section{Considerações Finais}

Em suma, os resultados obtidos neste estudo apontam para a complexidade da disfunção temporomandibular, uma vez que esta sofre influência de fatores variáveis, como foi exposto neste caso, no qual a paciente apresentou fatores etiológicos diversos que influenciam na fisiopatologia da disfunção. Dessa forma, o uso da laserterapia conseguiu ser eficaz na melhoria expressiva dos sinais e sintomas da paciente, bem como o auxílio da psicoterapia acentuou os resultados satisfatórios que foram obtidos. Por conseguinte, o uso dessa técnica pode trazer benefícios para os pacientes, porém se faz necessário maior divulgação e estudos científicos acerca do tema, com o objetivo de elucidar melhor a técnica e a padronização dos protocolos aplicados em casos de DTM de origem muscular.

\section{Referências}

Aparicio J. H., Delgado E. V., Domìnguez J. A., Tosti A. E. \& Escoda C.G. (2013). The use of low level laser therapy in the treatment of temporomandibular joint disorders. Review of the literature. Med Oral Patol Oral Cir Bucal. 18 (4): 603-612p.

Battistella C. B., Guimarães T. B., Quaglio C. L., Cabrini M. B. F, Martins D. A. G \& Novo N. F. (2016). Fatores biopsicossociais do Eixo II dos Critérios de Diagnóstico para Pesquisa das Desordens Temporomandibulares em indivíduos com disfunção temporomandibular muscular e migrânea. Rev Dor. São Paulo; 17(1):19-23p.

Bessa-Nogueira R. V., Vasconcelos B. C. \& Niederman R. (2008). The methodological quality of systematic reviews comparing temporomandibular joint disorder surgical and non-surgical treatment. BMC Oral Health. 26; 8:27p.

Carvalho A. A. F., Vieira J. B. \& Oliveira L. Q. C. (2014). Imagens tomográficas e radiográficas de forma incomum de cisto de retenção mucoso no seio maxilar direito. Rev Odontol UNESP, 43(N Especial):301p.

Castillo D. B., Azato F. K., Coelho T. K., Pereira P. Z \& Silva M. G. (2016). Estudo clínico da posição da cabeça e mandíbula em pacientes com disfunção temporomandibular muscular. Rev Dor. 17(2):88-92p.

Catão M. H. C. V., Oliveira P. S., Costa R. O. \& Carneiro V. S. M. (2012) Avaliação da eficácia do laser de baixa intensidade no tratamento das disfunções têmporo-mandibular: estudo clínico randomizado. Rev. CEFAC. 15(6): 1601-1608p.

Cavalcanti T. M., Almeida-Barros R. Q., Catão M. H. C. V., Feitosa A. P. A. \& Lins R. D. A. U. (2011). Conhecimento das propriedades físicas e da interação do laser com os tecidos biológicos na odontologia. An. Bras. Dermatol., Rio de Janeiro, 86 (5): $955-960$ p.

Dantas A. M. X., Santos E. J. L., Vilela R. M. \& Lucena L. B. S. (2015) Perfil epidemiológico de pacientes atendidos em um Serviço de Controle da Dor Orofacial. Rev Odontol UNESP. 44(6): 313-319.

Dias I. F. L. D., Siqueira C. P. C. M. S., Filho D. O. T., Duarte J. L., Laureto E. \& Lima F. M. (2009). Efeitos da luz em sistemas biológicos. Semina: Ciências Exatas e Tecnológicas. 30(1): 33-40p.

Dworkin S. F. \& LeRresche L. (1992). Research diagnostic criteria for temporomandibular disorders: review, criteria, examinations and specifications, critique. J Craniomandib Disord.6:301-5p.

Friedman M. H. (1993). Closed lock. A survey of 400 cases. Oral Surg Oral Med Oral Pathol. 75(4):422-7p.

Greenspan J. D. \& Winfield J.A. (1992). Reversible pain and tactile déficits associated with a cerebral tumor compressing the posterior insula and parietal operculum. Pain. 50(1): 29-39p. 
Research, Society and Development, v. 10, n. 6, e7810615390, 2021

(CC BY 4.0) | ISSN 2525-3409 | DOI: http://dx.doi.org/10.33448/rsd-v10i5.15390

Henriques A. C. G., Cazal C. \& Castro J. F. L. (2010). Ação da laserterapia no processo de proliferação e diferenciação celular. Revisão da literatura. Rev. Col. Bras. Cir. 37(4): 295-302p.

Lopez, V. J. (1986). El láser em tratamiento de las disfunciones de ATM. Rev Actual Estomatol Madrid. 46 (355):35-40p.

Ludke, M. \& Andre, M. E. D. A. (2013). Pesquisas em educação: uma abordagem qualitativa. E.P.U.

Mcneely M. L., Olivo S. A. \& Magee D. J. (2006). A systematic review of the effectiveness of physical therapy interventions for temporomandibular disorders. Phys Ther. 86(5):710-25p.

Piccin C. F., Pozzebon D., Chiodelli L., Boufleus J., Pasinato F. \& Corrêa E. C. R. (2016). Aspectos clínicos e psicossociais avaliados por critérios de diagnóstico para disfunção temporomandibular. Rev. CEFAC. 18(1):113-119p.

Pozzebon D., Piccin C. F., Silva A. M. T. \& Corrêa E. C. R. (2016). Disfunção temporomandibular e dor craniocervical em profissionais da área da enfermagem sob estresse no trabalho. Rev. CEFAC. 18(2):439-448p.

Quinto, C. A. (2000). Classificação e tratamento das disfunções temporomandibulares: qual o papel do fonoaudiólogo no tratamento dessas disfunções. Rev. Cefac, v.

Salmos-Brito J. A., de Menezes R. F., Teixeira C. E., Gonzaga R. K., Rodrigues B. H., Braz R., Bessa-Nogueira R. V. \& Gerbi M. E. (2013). Evaluation of low-level laser therapy in patients with acute and chronic temporomandibular disorders. Lasers Med Sci. 28(1):57-64p.

Venancio R. A., Camparis C. M. \& Lizarelli R. F. Z. (2002). Laser no Tratamento de Desordens Temporomandibulares. JBA, 2(7), 229-234.

Vera R. M. D. L. T., Grillo C. M., Fortinguerra M. L. B. Sousa M. L. R., \& Berzin F. (2013). Acupuntura no manuseio da dor orofacial e do tinido. Relato de caso. Rev Dor. 14(3):226-30p.

Webster G., Ikino C. M. Y., Salles B. W., Lino A. R., Manoel E. M. \& Filho W. C. (2011). Avaliação do efeito do tratamento de distúrbios temporomandibulares sobre o zumbido. Arq. Int. Otorrinolaringol. 15(3):327-332p. 